

\title{
Neutrino interaction physics and the DUNE Near Detector
}

\author{
Carneiro, M. F. ${ }^{a, *}$ \\ ${ }^{a}$ Brookhaven National Laboratory, \\ Upton, NY 11973, USA \\ E-mail: mcarneiro@bnl.gov
}

DUNE is a long-baseline neutrino oscillation experiment that will take data in a wideband neutrino beam at Fermilab, starting in the latter half of the 2020s. The experiment is planning to build a very capable near detector to facilitate the high precision extraction of oscillation parameters. Part of the mission of the near detector is to acquire powerful data sets that can be used to constrain the fits used in the oscillation analyses and improve the neutrino interaction model. In this talk, the importance and the potential of a vibrant program of neutrino interaction physics using this detector is described. A few case studies that illustrate the power of the DUNE near detector for studying neutrino interaction physics are described.

40th International Conference on High Energy physics - ICHEP2020

July 28 - August 6, 2020

Prague, Czech Republic (virtual meeting)

\footnotetext{
* Speaker for the DUNE Collaboration
} 


\section{Introduction}

Knowledge of neutrino-nucleus scattering cross sections is crucial to the global neutrino physics program. There is a good theoretical understanding of neutrino-nucleon interactions but we still have a long way to properly model the nuclear effects that define what we see in our detectors [1].

While many neutrino-nucleus interactions initially follow the well-understood mechanisms of neutrino-nucleon processes (quasi-elastic (CCQE) scattering, resonant pion production, deep inelastic scattering etc), hadrons produced by these interactions are susceptible to final-state interactions (FSI), which can completely change both the kinematics and composition of the final particle state detected. A charged pion produced in an initial resonant interaction can undergo different processes like charge exchange (changing the expected charge balance detected), elastic scattering (changing the transverse momentum information expected), or simply be absorbed and not be visible in the detector at all. The initial nuclear state has complex energy levels and multinucleon processes. This make it an oversimplification to simply treat the initial nucleus as a collection of independent nucleons, meaning that primary interactions between neutrinos and heavy nuclei are already more complicated than those with free nucleons.

It is critical to understand the nuclear environment to make accurate neutrino cross section predictions. As the importance of interference effects between primary interaction types, as well as the effects of final-state interactions, have become increasingly evident, we present a guideline for analyses based on final-state topology. to disentangle the contributions of these various complex effects.

\section{Effects on Oscillation and CP Sensitivity}

Cross sections and interaction models are directly used to reconstruct neutrino energy, which make the conversion of event rates into parameter measurements directly dependant on those models. To illustrate the point, consider the following outline concept of a two-detector, longbaseline oscillation analysis:

- reconstruct event topology and total event energy in the Near Detector;

- use a nuclear model and an interaction model to infer the neutrino interaction energy;

- use geometry differences and oscillation hypothesis to predict the neutrino spectrum at the Far Detector (FD);

- use the nuclear model and the estimated flux to reconstruct topology and energy in the FD;

- compare simulation and data and test your hypothesis.

One can easily see that the nuclear and interaction model information are used in several steps of the analysis process. If an incorrect nuclear model is assumed, the oscillation parameters extracted will be incorrect. The analysis process considers the uncertainty on the models allowing for the vast range of available, plausible models. That introduces large systematic uncertainties since different models give different values for the extracted parameters. Cross Section systematic errors are often the largest contribution to the error summary of neutrino oscillation experiments. 


\section{CP Violation Sensitivity}

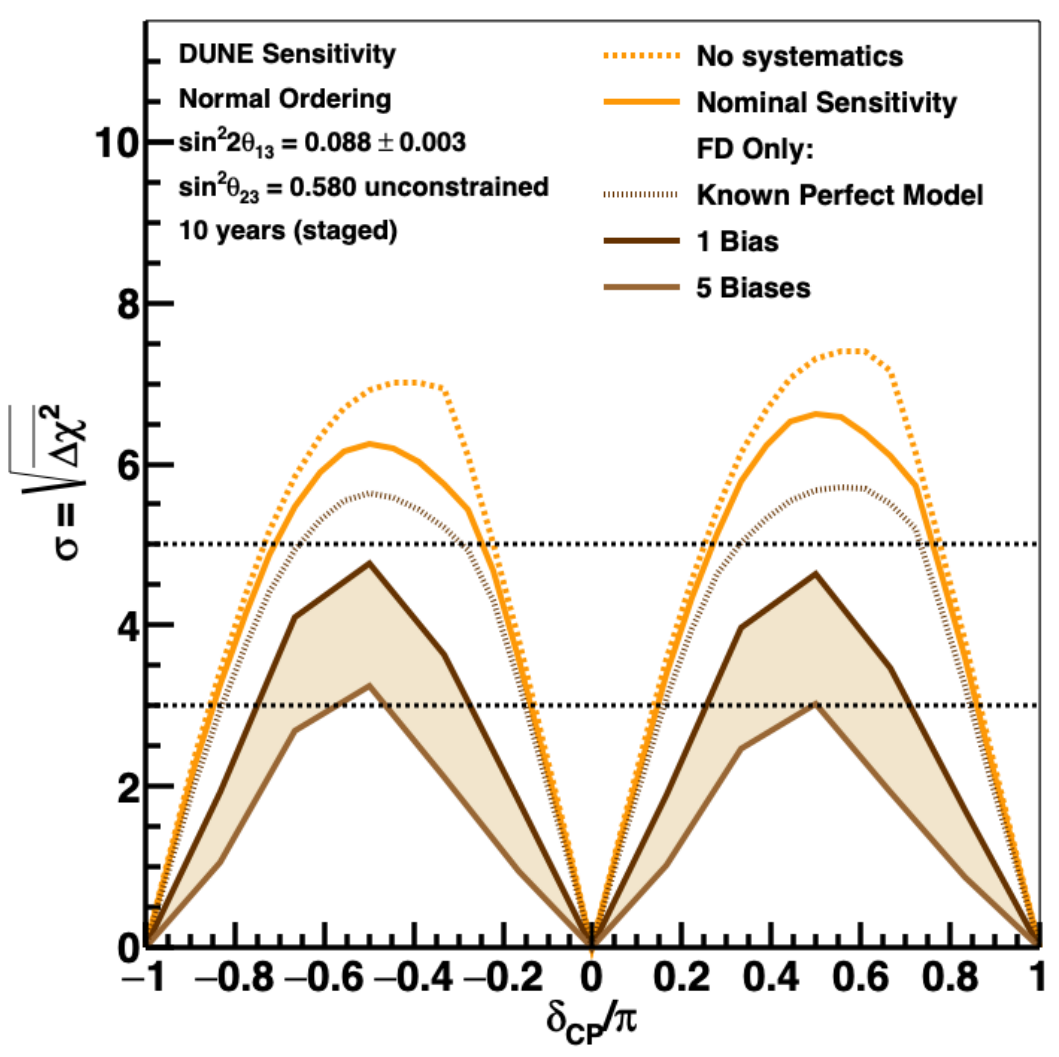

Figure 1: Effect on DUNE's sensitivity to the CP-violating phase if an incorrect cross section model is used in the analysis. This illustrates the danger of not improving/tuning the cross section model using data taken with the ND [2].

Eliminating incorrect models, as well as improving others, is necessary to reduce that uncertainty and thereby increase the precision of the oscillation parameters measured. The current level of precision we have in the ongoing neutrino oscillation program is a problem for the measurements we plan to do.

Nuclear models also play a big role in CP Violation Sensitivity. For this sensitivity we are dependent on the event generator assumptions of the cross section, but what if those assumptions are wrong? Fig. 1 shows a study that illustrates the situation. Initially a "known perfect model" is considered, meaning that the same model used to simulate the data was used to extract the sensitivity. The inclusion of a second, but plausible, model equires the addition of systematic errors that reduce the sensitivity. These are not the only possible viable models; more uncertainties due to model bias will need to be included, reducing sensitivity even further. A more through analysis of the study and results can be found at [3] 


\section{The Dune ND Complex}

The DUNE Near Detector (ND) complex is composed of 3 subsystems: the Near Detector Liquid Argon subdetector (ND-LAr), the Near Detector Gaseous Argon subdetector (ND-GAr) and the System for on-Axis Neutrino Detection (SAND) [4]. The ND-LAr detector has the same target nucleus and shares some aspects of form and functionality with the DUNE Far Detector (FD), with appropriate size to provide high statistics and hadron containment. The ND-GAr consists of a high-pressure gaseous argon time projection chamber (TPC) surrounded by an electromagnetic calorimeter (ECAL), providing a lower-density medium with a very low charged particle tracking threshold and excellent tracking resolution for muon detection. Muons are key to reconstructing charged-current neutrino interactions since the beam will primarily be composed of muon neutrinos, ND-GAr is also surrounded by a magnetic field to be able to differentiate $\mu^{+}$and $\mu^{-}$. It's position just upstream from ND-LAr allow the detection of high-energy, forward-going muons, increasing NDLAr's acceptance. SAND is a 3D projection scintillator tracker spectrometer, a plastic scintillator detector with an ECAL enclosed in a magnet serving as a consistent on-axis neutrino spectrum monitor. The ND-LAr and ND-GAr can move to take data in positions off the beam axis. This capability is referred to as DUNE-PRISM.

\section{Capabilities and Case Studies}

A rich spectrum of interaction types will take place in the near detector and we need the capability to analyse events based on the detected topology. Fig.2 shows the current simulation level expected distribution of events in the ND-GAr by pion multiplicity. Current simulation shows excellent particle identification and multiplicity classification capacity. Another important feature of the DUNE ND is the high statistics expected due to the proximity to the neutrino beam source. Table 1 show the expected statistics in ND-LAr and ND-GAr for 1 year of beam. For comparison the most recent MINERvA high statistics sample has $6 \times 10^{5}$ events [7].

\subsection{Separating interaction channels by pion multiplicity in ND-GAr}

Analysis of near-detector data will aim to extract samples corresponding to exclusive final states based on pion multiplicity. Due to nuclear effects and final-state interactions, each of these samples will contain contributions from various different nuclear interaction modes, meaning that predicted event rates will depend on multiple components of the interaction model. Comparing two different event generators we can see the spread on predictions resulting from the different viable models. Fig3 show the comparison between GENIE [5] and NuWro [6] event generators per multiplicity in the final state. Looking at each of these pion-multiplicity distributions and comparing data to generators' predictions will allow us to compare, select, and tune models reducing our viable model set and thus allowing us to achieve greater precision on oscillation parameter measurements.

\subsection{Investigating Nuclear Effects with Transverse Kinematic Imbalance}

Transverse Kinematic Imbalance (TKI) is a technique that helps precisely identify intranuclear dynamics [8-18] or the absence thereof [19-23] in neutrino-nucleus interactions. Applications of this technique in MINERvA's carbon hydrocarbon target [8-10] and T2K [16] show good agreement 


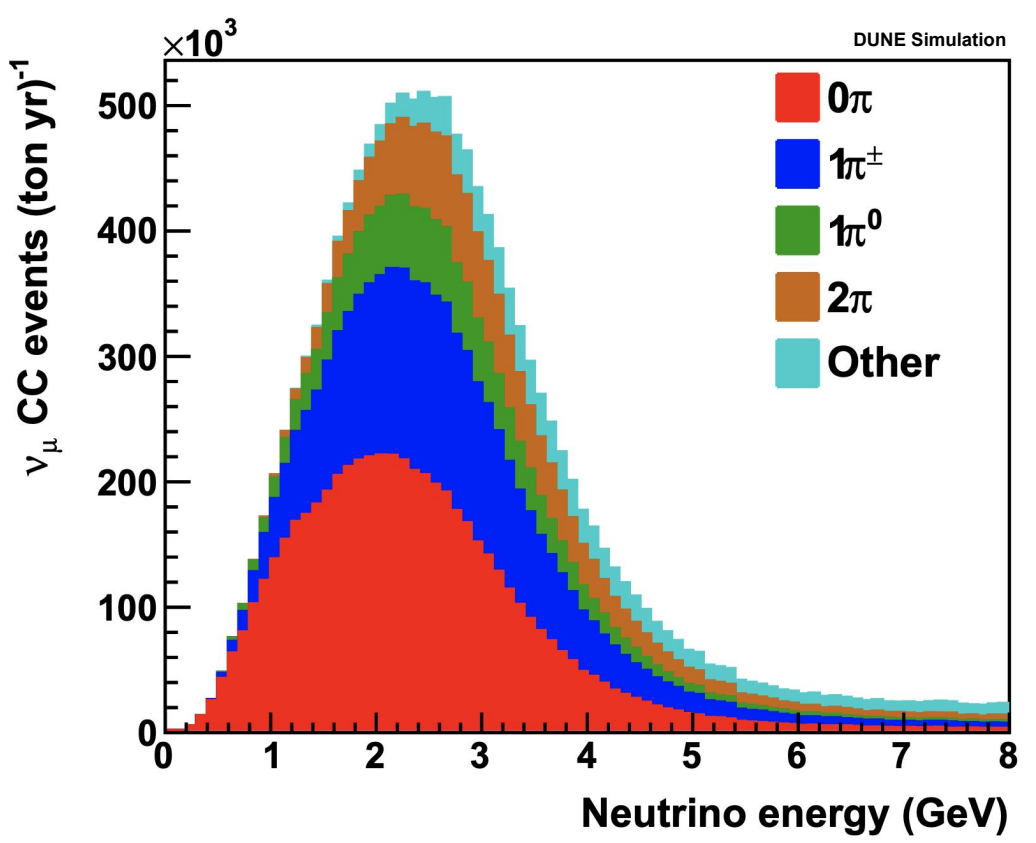

Figure 2: CC $v_{\mu}$ event rates on the argon by interaction type expected in the DUNE ND-GAr. The simulation corresponds to $1.97 \times 10^{21}$ protons on target in the forward horn current mode (one spill per second for a year) [2].

NuWro/GENIE for various reconstructed final states (FHC)

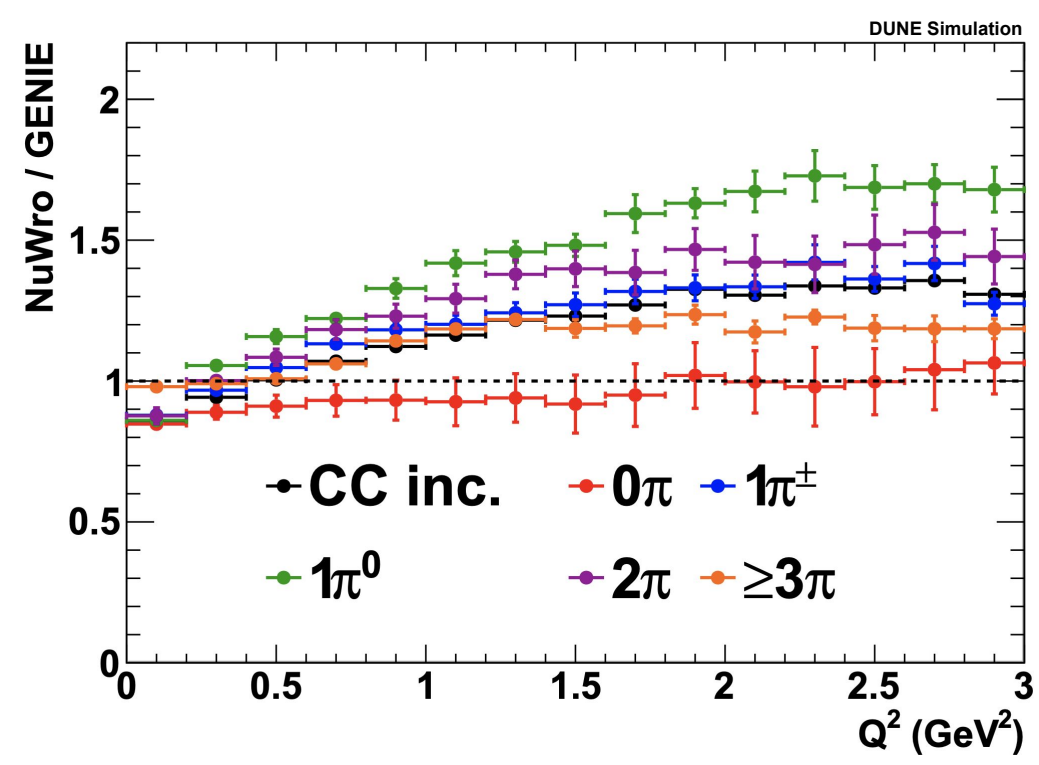

Figure 3: The ratio of the reconstructed $Q^{2}$ distributions for $v_{\mu}$ CC events for the NuWro and GENIE generators. Plots are shown for the ND-GAr, for final states including no pions (red), $1 \pi^{ \pm}$(blue), $1 \pi^{0}$ (green), 2 pions (purple) and 3 or more pions (orange) [2]. 
Table 1: Events per year $\left(1.1 \times 10^{21}\right.$ POT) in the forward horn-current $\left(v_{\mu}\right.$-favoring) mode. The rates were computed with GENIE 2.12.10. The rates assume a $50 t$ fiducial volume of liquid argon and a $1 t$ fiducial volume of argon gas [2].

\begin{tabular}{|c|c|c|c|c|c|}
\hline & \multicolumn{2}{|c|}{ Interaction Channel } & & \multicolumn{2}{|c|}{ Event Rate } \\
\hline & & & & ND-LAr & ND-GAr \\
\hline \multirow[t]{9}{*}{$\mathrm{CC}$} & $v_{\mu}$ & & & $8.2 \times 10^{7}$ & $1.64 \times 10^{6}$ \\
\hline & & $0 \pi$ & & $2.9 \times 10^{7}$ & $5.8 \times 10^{5}$ \\
\hline & & $1 \pi^{ \pm}$ & & $2.0 \times 10^{7}$ & $4.1 \times 10^{5}$ \\
\hline & & $1 \pi^{0}$ & & $8.1 \times 10^{6}$ & $1.6 \times 10^{5}$ \\
\hline & & $2 \pi$ & & $1.1 \times 10^{7}$ & $2.1 \times 10^{5}$ \\
\hline & & $3 \pi$ & & $4.6 \times 10^{6}$ & $9.3 \times 10^{4}$ \\
\hline & & other & & $9.2 \times 10^{6}$ & $1.8 \times 10^{5}$ \\
\hline & $\bar{v}_{\mu}$ & & & $3.6 \times 10^{6}$ & $7.1 \times 10^{4}$ \\
\hline & $v_{e}$ & & & $1.45 \times 10^{6}$ & $2.8 \times 10^{4}$ \\
\hline $\mathrm{NC}$ & & & & $5.3 \times 10^{5}$ & $5.5 \times 10^{5}$ \\
\hline$v+e$ & & & & $8.3 \times 10^{3}$ & $1.7 \times 10^{2}$ \\
\hline
\end{tabular}

with current implemented models but not in the transition region between Fermi motion and other nuclear effects. DUNE has a significantly larger phase space, with a full angular acceptance, and a great momentum acceptance. The excellent particle identification expected in all subsystems of DUNE ND will allow the use of TKI to investigate these effects in argon, shedding light on how these nuclear effects scale with nucleus composition.

\section{Conclusion}

Neutrino nucleus cross section knowledge is necessary for oscillation experiments and for neutrino physics as a whole. The DUNE ND not only provides near-far detector ratios to constrain flux uncertainty, it can also measure cross sections to constrain interaction models using argon and in the same neutrino beam as the FD. These measurements are not just necessary to help the oscillation measurement, but for a better understanding of nuclear physics, and of the nature of nuclear matter and how it behaves under the weak interaction. The DUNE ND physics program is vast and can provide a plethora of measurements and insights into the future of neutrino physics.

\section{References}

[1] L. Alvarez-Ruso et al. [NuSTEC],Prog. Part. Nucl. Phys. 100 (2018), 1-68 doi:10.1016/j.ppnp.2018.01.006 [arXiv:1706.03621 [hep-ph]].

[2] DUNE Near Detector Conceptual Design Report, in preparation

[3] B. Abi et al. [DUNE],[arXiv:2002.03005 [hep-ex]]. 
[4] B. Abi et al. [DUNE],JINST 15 (2020) no.08, T08008 doi:10.1088/1748-0221/15/08/T08008 [arXiv:2002.02967 [physics.ins-det]].

[5] C. Andreopoulos, A. Bell, D. Bhattacharya, F. Cavanna, J. Dobson, S. Dytman, H. Gallagher, P. Guzowski, R. Hatcher and P. Kehayias, et al. Nucl. Instrum. Meth. A 614 (2010), 87-104 doi:10.1016/j.nima.2009.12.009 [arXiv:0905.2517 [hep-ph]].

[6] T. Golan, J. T. Sobczyk and J. Zmuda, Nucl. Phys. B Proc. Suppl. 229-232 (2012), 499-499 doi:10.1016/j.nuclphysbps.2012.09.136

[7] M. F. Carneiro et al. [MINERvA], Phys. Rev. Lett. 124 (2020) no.12, 121801 doi:10.1103/PhysRevLett.124.121801 [arXiv:1912.09890 [hep-ex]].

[8] X. G. Lu et al. [MINERvA], Phys. Rev. Lett. 121 (2018) no.2, 022504 doi:10.1103/PhysRevLett.121.022504 [arXiv:1805.05486 [hep-ex]].

[9] T. Cai et al. [MINERvA], Phys. Rev. D $101 \quad$ (2020) no.9, 092001 doi:10.1103/PhysRevD.101.092001 [arXiv:1910.08658 [hep-ex]].

[10] D. Coplowe et al. [MINERvA], Phys. Rev. D 102, no.7, 072007 (2020) doi:10.1103/PhysRevD.102.072007 [arXiv:2002.05812 [hep-ex]].

[11] S. Dolan, [arXiv:1810.06043 [hep-ex]].

[12] T. Cai, X. Lu and D. Ruterbories, Phys. Rev. D 100, 073010 (2019) doi:10.1103/PhysRevD.100.073010 [arXiv:1907.11212 [hep-ex]].

[13] L. A. Harewood and R. Gran, [arXiv:1906.10576 [hep-ex]].

[14] X. Lu and J. T. Sobczyk, Phys. Rev. C 99, no.5, 055504 (2019) doi:10.1103/PhysRevC.99.055504 [arXiv:1901.06411 [hep-ph]].

[15] S. Dolan, U. Mosel, K. Gallmeister, L. Pickering and S. Bolognesi, Phys. Rev. C 98, no.4, 045502 (2018) doi:10.1103/PhysRevC.98.045502 [arXiv:1804.09488 [hep-ex]].

[16] K. Abe et al. [T2K], Phys. Rev. D 98, no.3, 032003 (2018) doi:10.1103/PhysRevD.98.032003 [arXiv:1802.05078 [hep-ex]].

[17] A. P. Furmanski and J. T. Sobczyk, Phys. Rev. C 95, no.6, 065501 (2017) doi:10.1103/PhysRevC.95.065501 [arXiv:1609.03530 [hep-ex]].

[18] X. G. Lu, L. Pickering, S. Dolan, G. Barr, D. Coplowe, Y. Uchida, D. Wark, M. O. Wascko, A. Weber and T. Yuan, Phys. Rev. C 94, no.1, 015503 (2016) doi:10.1103/PhysRevC.94.015503 [arXiv:1512.05748 [nucl-th]].

[19] P. Hamacher-Baumann, X. Lu and J. Martín-Albo, Phys. Rev. D 102, no.3, 033005 (2020) doi:10.1103/PhysRevD.102.033005 [arXiv:2005.05252 [physics.ins-det]]. 
[20] L. Munteanu, S. Suvorov, S. Dolan, D. Sgalaberna, S. Bolognesi, S. Manly, G. Yang, C. Giganti, K. Iwamoto and C. Jesús-Valls, Phys. Rev. D 101, no.9, 092003 (2020) doi:10.1103/PhysRevD.101.092003 [arXiv:1912.01511 [physics.ins-det]].

[21] H. Duyang, B. Guo, S. R. Mishra and R. Petti, Phys. Lett. B 795, 424-431 (2019) doi:10.1016/j.physletb.2019.06.003 [arXiv:1902.09480 [hep-ph]].

[22] H. Duyang, B. Guo, S. R. Mishra and R. Petti, [arXiv:1809.08752 [hep-ph]].

[23] X. G. Lu, D. Coplowe, R. Shah, G. Barr, D. Wark and A. Weber, Phys. Rev. D 92, no.5, 051302 (2015) doi:10.1103/PhysRevD.92.051302 [arXiv:1507.00967 [hep-ex]]. 